\title{
Extraction, purification and antibacterial activity of polysaccharides from Purslane
}

\author{
Chunmei Gu* 1, a , Shu Pan ${ }^{1, b}$ \\ ${ }^{1}$ Institute of Food Science and Engineering, Jilin Agricultural University, Changchun 130118, China \\ ajjnong2008@126.com, ${ }^{b} 269801095 @ q q . c o m$ \\ ${ }^{*}$ Corresponding author
}

\begin{abstract}
Keywords: Purslane polysaccharides, Purification, Antibacterial activity.
Abstract. In this study, the polysaccharides from Purslane were extracted by ultrasonic method and its content was measured by the phenol-sulfuric acid method using glucose as the standard. The crude polysaccharides were collected and purified by DEAE-Cellulose anion-exchange and Sephadex gel-permeation chromatography. Three fractions were obtained and named POL1, POL2 and POL3, respectively. The molecular weights of these three fractions were measured by Sephadex using dextran standard curve. Escherichia coli, Acetic acid bacteria, Saccharomycetes and Aspergillus Niger were used to study the antibacterial activity of the polysaccharides. The results showed that molecular weights of POL1, POL2 and POL 3 were $18 \mathrm{Kd}, 55 \mathrm{kd}$ and $108 \mathrm{kd}$, respectively. The polysaccharides had inhibitory effect on microorganisms, and the inhibited order of microorganisms was: Saccharomycetes>Acetic acid bacteria>Aspergillus Niger>Escherichia coli. In addition, the order of antibacterial activities of these three fractions on microorganisms was: POL3>POL2b>POL1b. It could be concluded that three fractions were obtained by purification. These three fractions had inhibitory effect on Saccharomycetes, Acetic acid bacteria, Aspergillus Niger and Escherichia coli. The antibacterial activity of polysaccharides from Purslane against Saccharomycetes was the strongest, while the weakest against Escherichia coli. In addition, POL3 was found to be the most effective against the tested microorganisms among the three fractions.
\end{abstract}

\section{Introduction}

Purslane is a nutritious vegetable used for human consumption [1] and can provide a rich plant source of nutritional benefits [2, 3]. It has been used as an antiseptic, and as an anti-diuretic for urinary disorders. A variety of research has shown that it exhibits a wide range of biological effects, including a skeletal muscle relaxant effect [4], analgesic and anti-inflammatory effects [5], antifungal activity [6] and an antifertility effect [7]. Purslane has also shown other beneficial effects such as antidiabetic [8] and wound healing properties [9]. Many studies have also shown that Purslane contains polysaccharides, flavonoids, coumarins, monoterpene glycoside and alkaloids [10]. Therefore, it is necessary to study on Purslane.

There has been an increasing interest in polysaccharides, mainly due to its biological activities. It has been reported that the polysaccharides have potential biological functions, for instance anti-tumor, anti-virus, immunity-modulation, antioxidation and so on [11]. Polysaccharide-containing extracts from plants, epiphytes and animals have been widely used for the treatment of some diseases in traditional Chinese medicine. Purslane is one of the interesting bioactive polysaccharides resources. Therefore, discovery and evaluation of new polysaccharides from Purslane has become a hot research spot. Several studies related to polysaccharides have drawn the attention of chemists and immunobiologists in recent years on account of their immunomodulation and anti-virus properties. Meanwhile, their antibacterial activities are worthy of further research $[12,13]$.

The main objectives of this work were to conduct a preliminary research on the extraction, purification and antibacterial effect of the polysaccharide in Purslane. This paper will contribute to understanding the characteristics and function of polysaccharides in Purslane and providing a foundation for the development of value-added products. 


\section{Materials and methods}

Material. Purslane was purchased from pharmacy in Changchun City, Jilin Province, China. The materials were shade-dried at room temperature. Other reagents were analytical grade as commercially available.

Extraction and purification of the polysaccharide. Dried Purslane was defatted in a Soxhlet apparatus with anhydrous ethanol to remove some coloured materials, monosaccharides, oligosaccharides and small molecule materials. The residue was then extracted with distilled water by ultrasonic at $60^{\circ} \mathrm{C}, 200 \mathrm{w}, 1: 20$ and $60 \mathrm{~min}$ for each time. After centrifugation, the supernatant was concentrated, and poured into 3 vol. of $95 \%$ ethanol for $24 \mathrm{~h}$ to precipitate the crude polysaccharides. After precipitation and drying, the crude polysaccharides were treated with Sevag reagent to remove proteins [14]. The process was repeated until there was no free protein in the supernatant, and then the supernatant was dialyzed against distilled water for $16 \mathrm{~h}$. Freezing dry of the dialyzate provided preliminarily purified polysaccharides.

Then the pretreated samples $(5 \mathrm{mg})$ were dissolved in distilled water $(10 \mathrm{~mL})$ and loaded on a DEAE-Cellulose column $(3.5 \mathrm{~cm} \times 20 \mathrm{~cm})$ preequilibrated with distilled water. The column was eluted first with distilled water at a flow rate of $0.5 \mathrm{~mL} / \mathrm{min}$, and then with aqueous $\mathrm{NaCl}$ solutions $(0.1 \mathrm{M}-0.6 \mathrm{M})$. Based on the results of this assay, the water-eluted fraction, $\mathrm{NaCl}$ eluted fraction were applied to a Sepharose $(1.6 \mathrm{~cm} \times 50 \mathrm{~cm})$ and eluted with $0.01 \mathrm{M} \mathrm{NaCl}$ at $0.5 \mathrm{~mL} / \mathrm{min}$, yielding purified polysaccharides.

Determination of polysaccharides content. The polysaccharides content was determined according to the phenol-sulfuric acid method [15]. Briefly, $1.0 \mathrm{~mL}$ of $5 \%$ phenol was added and mixed up. Then $5 \mathrm{~mL}$ of sulfuric acid was added and mixed. The absorbance of the solution was measured at $490 \mathrm{~nm}$ with an ultraviolet-visible spectrophotometer, using water as a blank.

Molecular weights of Purslane polysaccharides. The molecular weights of the polysaccharides fractions were determined by gel permeation chromatography (Sephadex G-100, $1.6 \mathrm{~cm} \times 50 \mathrm{~cm}$ ) [16]. The elution was carried out with $0.1 \mathrm{MNaCI}$ at a flow rate of $0.6 \mathrm{~mL} / \mathrm{min}$. Glucose and dextran standards with different molecular weights (T-10, T-40, T-70, T-500) were prepared using the same solvent. The equation of the standard curve was in Fig.1, log $\mathrm{Mw}=7.4958-1.5752 \mathrm{x}$ (where Mw represented the molecular weight, while $\mathrm{x}$ represented Ve/V) with a correlation coefficient of 0.994.

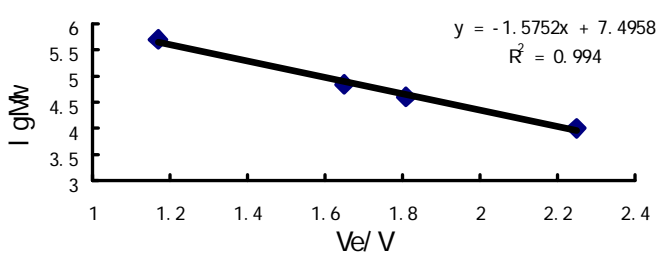

Figure 1: Dextran standard curve

Antibacterial activity of the polysaccharides. The antibacterial activity of the polysaccharides was studied by the method of paper disc diffusion assay with slight modification [17]. Escherichia coli, Acetic acid bacteria, Saccharomycetes and Aspergillus Niger were used as indication bacteria and inoculated in Luria Broth medium. With the inoculum solution $(100 \mu \mathrm{L})$ of each kind of microorganism smeared on the standard assay medium, paper disc with the polysaccharides solution $(5 \mathrm{mg} / \mathrm{mL})$ was placed in the medium. Sterilized physiological saline was used as a control. After the medium was incubated, the inhibition zones were measured.

Statistical analysis. SPSS 18.0 statistical software was used for data analysis. Data from different assay treatments were analyzed by one-way analysis of variance (ANOVA). Differences with $P<0.05$ were regarded as significant. 


\section{Results}

Extraction and purification of the polysaccharides. The crude polysaccharides with extraction yield of $35.42 \%$ of dried raw material, were obtained from Purslane by defatting, ultrasonic extraction, ethanol precipitation, deprotein by Sevag method, dialysis against water and freezing dry. Crude polysaccharides were further fractionated by DEAE-Cellulose anion-exchange and Sephadex gel-permeation chromatography. The elution profile of the polysaccharides on the DEAE-cellulose column was illustrated in Fig.2. The first peak was eluted with distilled water (POL1), and the other two peaks were eluted with $0.2 \mathrm{M} \mathrm{NaCl}$ (POL2) and $0.6 \mathrm{M} \mathrm{NaCl}$ (POL3), respectively.

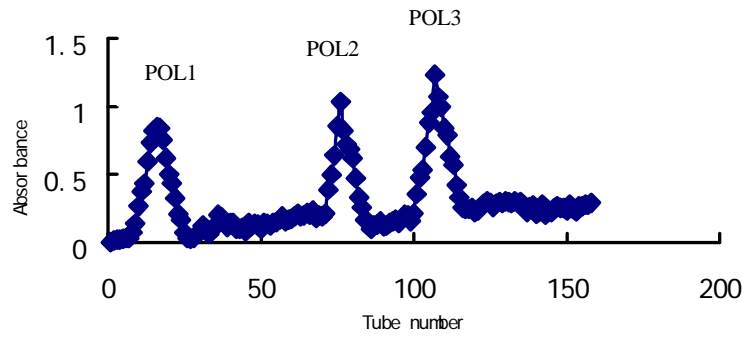

Figure 2 : Elution profiles of crude polysaccharide on DEAE-Cellulose

The three fractions were collected and applied to the Sepharose G-100 column, resulting in the elution profile in Fig.3, Fig.5 and Fig.7. Then the eluate by $0.01 \mathrm{M} \mathrm{NaCl}$ was collected.

In Fig.3, after POL1 was purified by Sepharose G-100, two peaks were obtained, and named POL1a and POL1b, respectively. The main peak was POL1b in Fig.3. So the POL1b was collected and applied to the Sepharose G-100 column again to determine whether it was a single peak. According to the result in Fig.4, POL1b was pure after purified by Sepharose G-100.

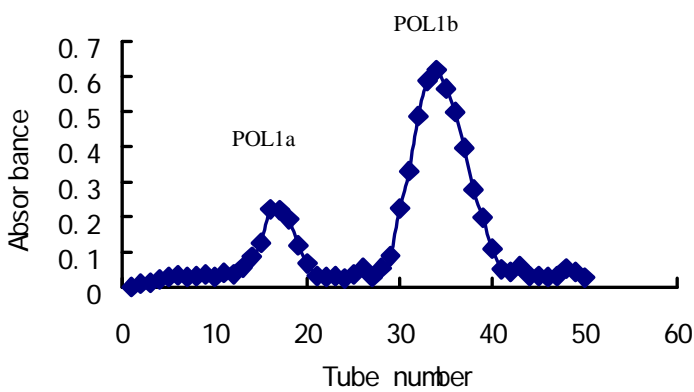

Figure 3 : Elution profiles of POL1 on Sepharose G-100

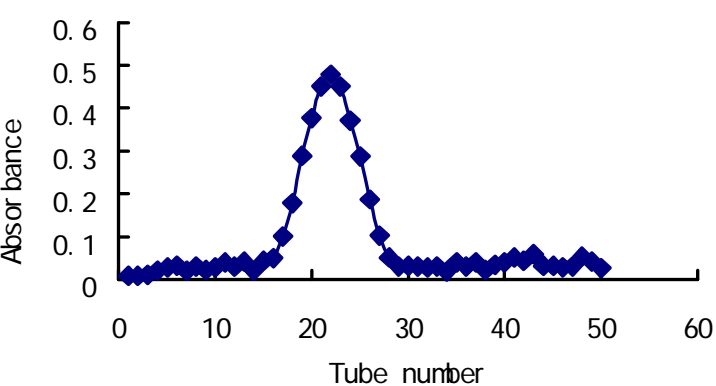

Figure 4 : Elution profiles of POL1b on Sepharose G-100

As can be seen in Fig. 5 there were two peaks, named POL2a and POL2b, respectively. The main peak was POL2b in Fig.5. So POL2b was collected and applied to the Sepharose G-100 column again to determine whether it was a single peak. According to the result in Fig.6, POL2b was pure after purified by Sepharose G-100.

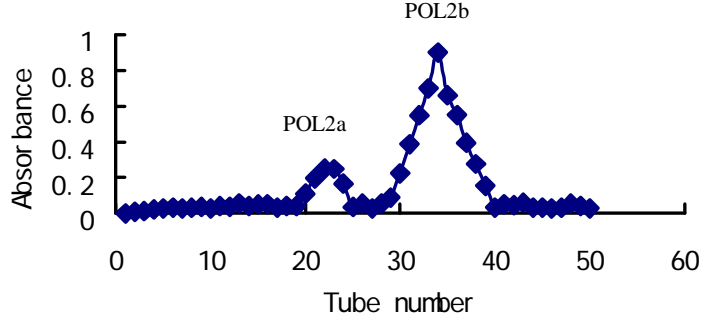

Figure 5: Elution profiles of POL2 on Sepharose G-100

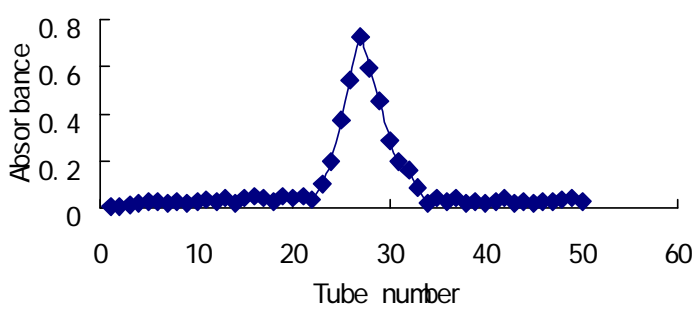

Figure 6: Elution profiles of POL2b on Sepharose G-100 


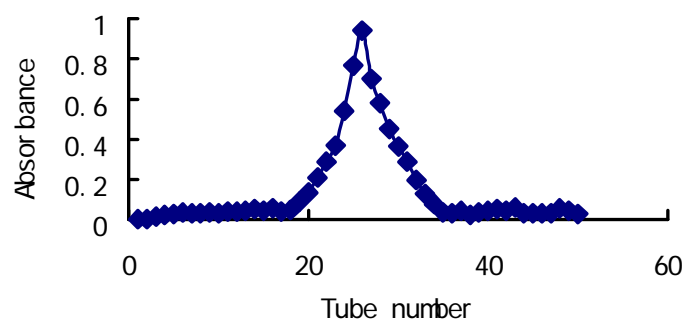

Figure 7: Elution profiles of POL3 on Sepharose G-100

Molecular weights of Purslane polysaccharides. The results of molecular weights were showed in Table 1. The molecular weight of the polysaccharides fractions were determined by gel permeation chromatography. According to the standard curve, the molecular weights of the three polysaccharide fractions POL1b, POL2b and POL3 were found to be 18,55 and $108 \mathrm{kDa}$, respectively.

Table 1. The molecular weights of polysaccharides

\begin{tabular}{cc}
\hline Polysaccharide fractions & Molecular weights \\
\hline POL1b & $18 \mathrm{kDa}$ \\
POL2b & $55 \mathrm{kDa}$ \\
POL3 & $108 \mathrm{kDa}$ \\
\hline
\end{tabular}

Antibacterial activity of the polysaccharides. In vitro antibacterial activities of the polysaccharides were shown in Table 2. According to the data, the polysaccharides had inhibitory effect on these microorganisms. POL3 was found to be the most effective against the tested microorganisms with the inhibition zones of $16.33 \mathrm{~mm}$ (Saccharomycetes), $15.10 \mathrm{~mm}$ (Acetic acid bacteria), $14.20 \mathrm{~mm}$ (Aspergillus Niger ) and 12.93mm (Escherichia coli ) among the three fractions. The antibacterial activities of POL2b were better than that of POL1b, with the inhibition zones of $15.40 \mathrm{~mm}$ (Saccharomycetes), $14.03 \mathrm{~mm}$ (Acetic acid bacteria), 12.60mm (Aspergillus Niger) and $11.17 \mathrm{~mm}$ (Escherichia coli). The antibacterial activities of POL1b were weakest in these three fractions, with the inhibition zone of $14.03 \mathrm{~mm}$ (Saccharomycetes), $13.17 \mathrm{~mm}$ (Acetic acid bacteria), $11.97 \mathrm{~mm}$ (Aspergillus Niger) and $10.40 \mathrm{~mm}$ (Escherichia coli). According to the above results, the antibacterial activity of polysaccharides from Purslane against Saccharomycetes was the strongest, while the weakest against Escherichia coli. The inhibited order of microorganisms was: Saccharomycetes $>$ Acetic acid bacteria $>$ Aspergillus Niger $>$ Escherichia coli.

Table 2. The antibacterial activities of POL1b, POL2b and POL3

\begin{tabular}{ccccc}
\hline \multirow{2}{*}{$\begin{array}{c}\text { Polysaccharide } \\
\text { fractions }\end{array}$} & Saccharomycetes & $\begin{array}{c}\text { Acetic acid } \\
\text { bacteria }\end{array}$ & Aspergillus Niger & Escherichia coli \\
\cline { 2 - 5 } & $14.03 \pm 0.50^{\mathrm{a}}$ & $13.17 \pm 0.42^{\mathrm{a}}$ & $11.97 \pm 0.45^{\mathrm{a}}$ & $10.40 \pm 0.36^{\mathrm{a}}$ \\
\hline POL1b & $15.40 \pm 0.25^{\mathrm{b}}$ & $14.03 \pm 0.45^{\mathrm{b}}$ & $12.60 \pm 0.17^{\mathrm{a}}$ & $11.17 \pm 0.32^{\mathrm{a}}$ \\
POL2b & $16.33 \pm 0.29^{\mathrm{c}}$ & $15.10 \pm 0.36^{\mathrm{c}}$ & $14.20 \pm 0.35^{\mathrm{b}}$ & $12.93 \pm 0.51^{\mathrm{b}}$ \\
\hline
\end{tabular}

All data are the mean and standard deviation of three determinations.

Column data marked with different superscripts mean significant difference $(P<0.05)$; with same superscripts mean no significant difference $(P>0.05)$. 


\section{Discussion}

In recent years, biological activities of polysaccharides from Purslane have been studied, such as antibacterial, anti-tumor, anti-virus and so on. Meanwhile, the composition and molecular weights of polysaccharides also have been studied [18]. In this study, the polysaccharides were extracted from Purslane by ultrasonic method and purified by DEAE-Cellulose anion-exchange and Sephadex gel-permeation chromatography. Three fractions were obtained and named POL1, POL2 and POL3, with molecular weights of $18 \mathrm{Kd}, 55 \mathrm{kd}$ and $108 \mathrm{kd}$, respectively, which were different from Shen's report that the molecular weight of polysaccharides from Purslane was $26 \mathrm{kDa}$ by the method of gel permeation chromatography [19]. The possible reasons for the molecular weight difference of polysaccharides were, on the one hand, the source of Purslane used in the study and the kind of polysaccharides were different; on the other hand, the eluent is different during purification.

It was reported [20] that polysaccharides from Purslane had antibacterial activities, which was coincident with our results. In our study, the polysaccharide from Purslane showed the antibacterial effect on the tested microorganisms (displayed in Table 2). Moreover, it has been reported that the antibacterial activity of polysaccharide might depend on its monosaccharide composition, molecular weight and structure of the polymeric backbone [21-23]. The relatively higher molecular mass seemed to increase the antibacterial activities [24]. Our data showed that the molecular weight distribution of POL3 was 108kd and higher than that of POL1b and POL2b (shown in Table 1), moreover, the antibacterial activity of POL3 was stronger than other two fractions and the antibacterial effect on the microorganisms was the most effective. This result accorded with the above theory, that is, the higher molecular weight, the stronger antibacterial activity. Many reports showed that polysaccharides had the antibacterial ability. For example, Zhu et al. reported that polysaccharides had inhibitory effect on Escherichia coli, Staphylococcus aureus and Sarcina lutea [25]. He et al. found that polysaccharides could inhibit Staphylococcus aureus, Bacillus subtilis, Listeria monocytogenes, Escherichia coli, Zygosaccharomyces bailii and Candida utilis [26]. Wang et al. reported that polysaccharides had inhibitory effect on Escherichia coli, Bacillus subtilis and Staphylococcus aureus [27]. In spite of extensive studies on the antibacterial activity of polysaccharides, the inhibitory effect of polysaccharides from Purslane on Saccharomycetes, Acetic acid bacteria, Aspergillus Niger has rarely been studied. Li et al. reported that the inhibition zone of polysaccharides from Pleurotus eryngii on Escherichia coli was $11.7 \mathrm{~mm}$ [28]. However, in our study, the inhibition zone of polysaccharides from Purslane on Escherichia coli was $12.93 \mathrm{~mm}$. Gao et al. reported that the inhibition zone of polysaccharides from Chinese wolfberry on Aspergillus Niger was 22.25mm [29]. But, in our study, the inhibition zone of polysaccharides from Purslane on Aspergillus Niger was 14.20. According to the above results, the differences of antibacterial activity of polysaccharides may be related to the sources of polysaccharides, types and concentration. In this study, all these three fractions showed higher antibacterial activities on Saccharomycetes. The order of antibacterial activities on microorganisms was: Saccharomycetes $>$ Acetic acid bacteria $>$ Aspergillus Niger $>$ Escherichia coli. Due to the complex relationship of the native structure, conformation and biological functions, the mechanisms of bioactivities found in the polysaccharide still need further to be studied.

It is significant to search clues to possible antibacterial mechanisms of the polysaccharide for comprehensive utilization of Purslane. Based on these results, it was speculated that the polysaccharide in Purslane could play a significant role in the prevention of food spoilage and food poisoning or medical treatment.

\section{Conclusions}

The crude polysaccharides were purified by DEAE-Cellulose anion-exchange and Sepharose gel-permeation chromatography and three fractions were obtained, with molecular weights of $18 \mathrm{Kd}$, $55 \mathrm{Kd}$ and $108 \mathrm{Kd}$, respectively. POL3 was found to be more effective against the tested microorganisms than other fractions. The antibacterial activity of polysaccharides from Purslane 
against Saccharomycetes was the strongest, while the weakest against Escherichia coli. Our results showed that polysaccharides had antibacterial activity and were a viable option for using as food preservative.

\section{References}

[1] A. I. Mohamed, A. S. Hussein, Chemical composition of purslane (Portulaca oleracea), Plant Foods Hum. Nutr. 45 (1994) 1-9.

[2] D. Sudhakar, R. K. Kishore, P. R. Parthasarathy, Portulaca oleracea L. extract ameliorates the cisplatin-induced toxicity in chick embryonic liver, Indian J. Biochem. Biophys. 47 (2010) 185-189.

[3] I. Oliveira, P. Valent, R. Lopes, P. B. Andrade, A. Bento, J. A. Pereira, Phytochemical characterization and radical scavenging activity of Portulaca oleraceae L. leaves and stems, Microchem. J. 92 (2009) 129-134

[4] O. Parry, J. A. Marks, F. K. Okwuasaba, The skeletal muscle relaxant action of Portulaca oleracea: role of potassium ions, J. Ethnopharmacol 40 (1993) 187-194.

[5] K. Chan, M. W. Islam, M. Kamil, R. Radhakrishnan, M. N. Zakaria, M. Habibullah, A. Attas, The analgesic and anti-inflammatory effects of Portulaca oleracea L.subsp. Sativa (Haw.) Celak, J. Ethnopharmacol. 73 (2000) 445-451.

[6] V. Suresh, N. Senthilkumar, R. Thangam, M. Rajkumar, C. Anbazhagana, R. Rengasamy, P. Gunasekaran, S. Kannand, P. Palani, Separation, purification and preliminary characterization of sulfated polysaccharides from Sargassum plagiophyllum and its in vitro anticancer and antioxidant activity, Process Biochemistry 48 (2013) 364-373.

[7] N. E. Awad, Lipid content and antimicrobial activity of phenolic constituents of cultivated Portulaca oleracea L., Bulletin of Faculty of Pharmacy 32 (1994) 137-142.

[8] M. A. Dkhil, A. E. Abdel Moniem, S. Al-Quraishy, R. A. Saleh, Antioxidant effect of purslane (Portulaca oleracea) and its mechanism of action, J.Med. Plant Res. 5 (2011) 1563-1589.

[9] A. N. Rashed, F. U. Afifi, A. M. Disi, Simple evaluation of the wound healing activity of a crude extract of Portulaca oleracea L. (growing in Jordan) in Mus musculus JVI-1, J. Ethnopharmacol. 88 (2003) 131-136.

[10] T. B. Mohammad, H. B. Mohammad, M. Farhad, Antitussive effect of Portulaca oleracea L. in guinea pigs, Iran. J. Pharm. Res. 3 (2004) 187-190.

[11] Y. X. Lei, N. Zhong, M. Yang, B. Zhu, Anti-tumor activity of Chlamys farrefi glycoprotein and polysaccharides on S180 Mice, China Pharmacist 9(2) (2006) 123-124.

[12]J. B. Xiao, J. L. Huo, H. X. Jiang, F. Yang, Chemical compositions and bioactivities of crude polysaccharides from tea leaves beyond their useful date, Int. J. Biol. Macromol. 49 (2011) 1143-1151.

[13]X. J. Zhang, Y. B. Ji, Z. Y. Qu, J. C. Xia, L. Wang, Experimental studies on antibiotic functions of Portulaca oleracea L. in vitro, Chinese Journal of Microbiology, 14 (2002) 277-280.

[14]M. G. Sevag, D. B. Lackman, J. Smolens, The isolation of the components of streptococcal nucleoproteins in serologically active form, J. Biol. Chem. 124 (1938) 425-436.

[15]H. Y. Zhang, L. Zheng, The extraction of purslane polysaccharide and antibacterial activity in vitro, Jiangsu agricultural science 39 (2011) 413-415. 
[16] J. H. Xie, M. Y. Xie, M. Y. Shen, S. P. Nie, Y. X. Wang, C. Li, Optimisationof microwave-assisted extraction of polysaccharides from Cyclocarya paliurus (Batal.) Iljinskaja using response surface methodology, J. Sci Food Agric. 90 (8) (2010) 1353-1360.

[17]H. J. Zhu, K. Sheng, E. F. Yan, J. J. Qiao, F. Lv, Extraction, purification and antibacterial activities of a polysaccharide from spent mushroom substrate, Int. J. Biol. Macromol. 50 (2012) 840-843.

[18] T. Chen, L. Zhu, X. Y. Liu, Y. Y. Li, C. D. Zhao, Z. G. Xu, W. F. Yan, H. X. Zhang, Synthesis and antioxidant activity of phosphorylated polysaccharide from Portulaca oleracea L. with $\mathrm{H}_{3} \mathrm{PW}_{12} \mathrm{O}_{40}$ immobilized on polyamine functionalized polystyrene bead as catalyst, J. Mol. Catal. A Chem. 342 (2011) 74-82.

[19]H. Shen, G. Tang, G. Zeng, Y. Yang, X. W. Cai, D. G. Li, H. C. Liu, N. X. Zhou, Purification and characterization of an antitumor polysaccharide from Portulaca oleracea L., Carbohydr. Polym. 93 (2013) 395-400.

[20] Y. Z. Tao, Y. Y. Zhang, L. N. Zhang, Chemical modification and antitumor activities of two polysaccharide-protein complexes from Pleurotus tuberregium, Int. J. Biol. Macromol. 45 (2009) $109-115$.

[21]F. J. Cui, W. Y. Tao, Z. H. Xu, W. J. Guo, H. Y. Xu, Z. H. Ao, J. Jin, Y. Q. Wei, Structural analysis of antitumor heteropolysaccharide GFPS1b from the cultured mycelia of Grifola frondosa GF9801, Bioresour. Technol. 98 (2007) 395-401.

[22]L. Y. Zhao, Y. H. Dong, G. T. Chen, Q. H. Hu, Extraction, purification, characterization and antitumor activity of polysaccharides from Ganoderma lucidum, Carbohydr. Polym. 80 (2010) 783-789.

[23]H. Wu, H. W. Guo, R. Zhao, Effect of lycium barbarum polysaccharide on the improvement of antioxidant ability and DNA Damage in NIDDM rats, Yakugaku Zasshi 126 (2006) 365-371.

[24] S. C. Chang, B. Y. Hsu, B. H. Chen, Structural characterization of polysaccharides from Zizyphus jujuba and evaluation of antioxidant activity, Int. J. Biol. Macromol. 47 (2010) 445-453.

[25]H. J. Zhu, K. Sheng, E. F. Yan, J. J. Qiao, F. Lv, Extraction, purification and antibacterial activities of a polysaccharide from spent mushroom substrate, Int. J. Biol. Macromol. 50 (2012) 840-843.

[26]F. He, Y. Yang, G. Yang, L. J. Yu, Studies on antibacterial activity and antibacterial mechanism of a nove polysaccharide from Streptomyces virginia H03, Food Control 21 (2010) 1257-1262.

[27]H. B. Wang, Cellulase-assisted extraction and antibacterial activity of polysaccharides from the dandelion Taraxacum officinale, Carbohydr. Polym. 103 (2014) 140-142.

[28] S. Q. Li, N. P. Shah, Antioxidant and antibacterial activities of sulphated polysaccharides from Pleurotus eryngii and Streptococcus thermophilus ASCC 1275, Food Chem. 165 (2014) 262-270.

[29]C. Y. Gao, L. Z. Jiang, C. R. Tian, Study on the antibacterial activity of Ch. Wolfberry polysaccharide, Food Sci. Technol. 10 (2007) 100-102. 